\title{
Atlas of human parasitology
}

By L. R. Ash and T. C. Orihel. 1984. 2nd edn. Raven Press, New York. Pp. xi and 212. US\$38.50.

This book is intended primarily for use in the laboratory. Almost all the photographs were prepared from clinical materials processed by standard diagnostic and clinical laboratory procedures.

Part $\mathrm{I}$ is devoted to diagnostic procedures and parasite identification. The use of good microscope techniques and the value of precise measurements in diagnostic parasitology are emphasised, the size of organisms often being a significant factor in differential diagnosis. The characteristic structure of protozoa and helminths and the useful features for their diagnosis are summarized. Part II contains the Atlas. The figures on each plate are well described and the illustrations are accompanied by limited, descriptive texts on the classification, disease, geographic distribution, location in host, morphology, life cycle and diagnosis for each organism.

Most of the intestinal protozoa shown in the Atlas have been photographed in faecal smears stained with trichrome or iron haematoxylin but some are illustrated as they appear in temporary wet mount preparations stained with iodine or unstained. The stain of choice for the blood and tissue protozoa is Giemsa's stain, whereas both Giemsa's and haematoxylin stains have been used for microfilariae in blood smears. The photographs of helminth eggs and larvae are as they appear in saline in iodine-stained temporary wet mounts. The sizes of parasiteelements are not provided with each illustration, but the measurements, including ranges and means, are found in the text description of each parasite, as well as in summary tables. All the malaria and Babesia organisms were photographed under oil immersion at one magnification on the same microscope, to assist comparison of parasite size and morphology. With few exceptions, the illustrations are of human material, animal specimens being used only when the parasite is difficult to obtain from human sources, e.g., trypanosomes. The diagnostic stages of animal parasites that are also important in man have been included.

The bibliography comprises 24 key references in medical parasitology, tropical medicine and general parasitology, and six references to other manuals and teaching aids. There is also a useful index.

The illustrations are, with few exceptions, well reproduced, but more use of arrows indicating key features might have been helpful. The inclusion of human malarial, as well as Babesia and other protozoal, parasites in this second edition is welcome. The Atlas now offers a comprehensive pictorial record of the principal human parasites, and is a valuable addition to laboratory diagnostic literature.

G. WeBBE 